Check for updates

Cite this: RSC Adv., 2018, 8, 40564

Received 8th October 2018

Accepted 26th November 2018

DOI: $10.1039 / c 8 r a 08296 k$

rsc.li/rsc-advances

\section{Detection of nucleic acids via G-quadruplex- controlled L-cysteine oxidation and catalyzed hairpin assembly-assisted signal amplification $\uparrow$}

\author{
Piaopiao Chen, ${ }^{a}$ Pingyue Hu, ${ }^{b}$ Ke Huang, (D) ${ }^{b}$ Erica Sawyer, ${ }^{a}$ Ke Sun, ${ }^{a}$ Binwu Ying, ${ }^{a}$ \\ Xiawei Wei ${ }^{a}$ and Jia Geng (iD *a
}

\begin{abstract}
The development of simple, sensitive and cost-effective methods for specific nucleic acid detection has attracted tremendous attention due to its importance to the early diagnosis of genetic diseases and to biodefense applications. In this work, we demonstrated a fluorescent turn-off mode DNA assay based on L-cysteine-modulated synthesis of CdTe quantum dots (CdTe QDs), horseradish peroxidase-mimicking G-quadruplex-hemin- $\mathrm{K}^{+}$complex controlled oxidation of L-cysteine to cystine, and catalyzed hairpin assembly (CHA)-assisted signal amplification. After the addition of target DNA, the CHA signal amplification reaction was triggered and numerous $\mathrm{H} 1-\mathrm{H} 2$ double-stranded DNA were formed, initiating the release of $\mathrm{G}$-quadruplex sequences in $\mathrm{H} 2$ simultaneously. Thus, the degree of inhibition of the synthesis of CdTe QDs is proportional to the concentration of the G-quadruplex sequence in this method. In contrast, when the target DNA was absent, the CHA could not be triggered, and the fluorescence signal was high due to the remaining intact L-cysteine. Under optimal experimental conditions, the homogeneous fluorescence method achieved the detection of HIV DNA with a linear range from $0.1 \mathrm{pM}$ to $1 \mathrm{nM}$ and a detection limit of $0.12 \mathrm{pM}$. This novel biosensor exhibits excellent specificity in differentiating DNA sequences with a single-base and two-base mismatch. To the best of our knowledge, this a label-free and highly sensitive bioassay utilizing CHA-assisted signal amplification and G-quadruplex control of in situ synthesis of CdTe QDs strategy was not reported in previous. Thus, this proposed strategy is anticipated to find use in basic biochemical research and clinical diagnosis.
\end{abstract}

\section{Introduction}

Recently, sequence-specific nucleic acid detection has received more and more attention due to its extreme importance in the clinical molecular diagnosis of diseases, biomedical studies, and cancer therapy. ${ }^{\mathbf{1 , 2}}$ A variety of sensitive approaches have been developed, such as colorimetric, ${ }^{3}$ fluorescent, ${ }^{4}$ chemiluminescence (CL), ${ }^{5}$ electrochemiluminescence (ECL), ${ }^{6}$ electrochemical, ${ }^{7}$ surface enhanced Raman spectroscopy (SERS), ${ }^{8}$ quartz crystal microbalance (QCM), ${ }^{9}$ atomic fluorescence spectrometry (AFS), ${ }^{10}$ inductively coupled plasma mass spectrometry (ICP-MS) methods, ${ }^{11}$ and nanopores etc. ${ }^{12}$ Among these sensing methods, the fluorescence-based is one of the most powerful and widely used approaches because of its rapidity, simplicity, high sensitivity, and accessible instrument requirements.

\footnotetext{
${ }^{a}$ Department of Laboratory Medicine, State Key Laboratory of Biotherapy, West China Hospital, Sichuan University and Collaborative Innovation Center for Biotherapy, Chengdu, Sichuan, 610041, China.E-mail: geng.jia@scu.edn.cn

${ }^{b}$ College of Chemistry and Material Science, Sichuan Normal University, Chengdu, Sichuan, 610068, China

$\dagger$ Electronic supplementary information (ESI) available. See DOI: 10.1039/c8ra08296k
}

Fluorescent inorganic nanoparticles have been generously synthesized by biomineralization processes, in which biomolecules are used as templates to mediate the formation of fluorescent nanoparticles. ${ }^{13}$ Among these biomineralized fluorescent inorganic metal nanoparticles, quantum dots (QDs) exhibit unique photophysical properties, such as high fluorescence quantum yields, large Stokes shifts, narrow and symmetric emission spectra, optically tunable light emissions, and superior stability against photobleaching. QDs have potential applications in numerous fields, such as sensing, bioimaging, and disease diagnosis, etc. ${ }^{\mathbf{1 4 - 1 6}}$ Also well known, Lcysteine could serve as an excellent ligand for CdTe QDs growths. ${ }^{17}$ The G-quadruplex-hemin- $\mathrm{K}^{+}$complex DNAzyme, which mimics horseradish peroxidase, has attracted substantial interest in the past decade due to its catalytic ability to oxidize various substrates, such as $\mathrm{ABTS}^{2-}$ and L-cysteine. These properties were extensively used to develop optical sensors for DNA, ${ }^{18}$ aptamer-substrate complexes, ${ }^{19}$ and small molecule. ${ }^{20}$ Therefore, through a combination of the L-cysteine-mediated synthesis of quantum dots and the catalytic oxidation of $\mathrm{L}^{-}$ cysteine to cystine (thiols to disulfides) by the G-quadruplexhemin $-\mathrm{K}^{+}$complex, fluorescent bioanalysis may be feasible. 
Until now, various signal amplification strategies have been pursued to explore ultrasensitive detection capability, which can be classified into enzyme-assisted and enzyme-free approaches. The enzyme-assisted approach signal amplification, such as polymerase, ligase, nicking endonuclease, exonuclease, and double-strand specific nuclease. ${ }^{21-24}$ Although target cycling methods via nucleases are highly sensitive and selective, the detection cost increase greatly and applications may be limited. Compared to the enzyme-assisted approach, enzyme-free signal amplification, such as hybridization chain reaction (HCR), ${ }^{25}$ strand displacement amplification (SDA), ${ }^{26}$ and catalyzed hairpin assembly (CHA), ${ }^{27}$ possesses unique advantages in detection due to its simple, robust, and low-cost features. Among the signal amplification methods, CHA signal amplification is an isothermal amplification reaction, and it utilizes a toehold-mediated strand displacement mechanism to continuously assemble DNA duplexes and translate the input of nucleic acid targets into an amplified signal with a low detection limit. ${ }^{28}$

To further extend the range of applications of biomimetic synthesis of luminescent nanomaterials and improve their analytical performance, a simple, homogeneous fluorescent turn-off nanoplatform by taking full advantage of G-quadruplex-hemin- $\mathrm{K}^{+}$-catalyzed oxidation reaction, L-cysteinemediated synthesis of fluorescent QDs and CHA nucleic acid amplification technique was developed for HIV DNA assay in this work. Given the high signal amplification efficiency of CHA, and the intrinsically high sensitivity of fluorescence detection, the proposed method provides a simple and highly sensitive platform for nucleic acid assay with a low detection limit.

\section{Experimental section}

\section{Materials}

All reagents used were of the highest purity available but at least of analytical grade and used without further purification. High purity sodium nitrate $\left(\mathrm{NaNO}_{3}\right)$, sodium hydroxide $(\mathrm{NaOH})$, potassium chloride $(\mathrm{KCl})$, cadmium chloride $\left(\mathrm{CdCl}_{2} \cdot 2.5 \mathrm{H}_{2} \mathrm{O}\right)$, sodium tellurite $\left(\mathrm{Na}_{2} \mathrm{TeO}_{3}\right)$ and sodium borohydride $\left(\mathrm{NaBH}_{4}\right)$ were purchased from Kelong Chemical Reagents (Chengdu, China). Hemin and 3-( $N$-morpholino) propanesulfonic acid (MOPS) were purchased from Solarbio Technology Co., Ltd. (Beijing, China). Dimethyl sulfoxide (DMSO) was obtained from Sigma-Aldrich (St. Louis, MO, USA.). L-Cysteine was obtained from Sinopharm Chemical Reagent Co., Ltd. (Beijing, China). All oligonucleotides were synthesized and purified by Sangon Biotechnology Co. Ltd. (Shanghai, China). The sequences of the employed DNA oligonucleotides are shown in Table 1. Human serum samples were provided by the Department of Laboratory Medicine, West China Hospital of Sichuan University (Chengdu, China). All work solutions were prepared with MOPS buffer solution (10 mM, $150 \mathrm{mM} \mathrm{NaNO}_{3}, \mathrm{pH}$ 7.6). High purity deionized water $(18.2 \mathrm{M} \Omega \mathrm{cm})$ from a water purification system (Chengdu Ultrapure Technology Co., Ltd., Chengdu, China) was used throughout this work. All solutions were stored at $4{ }^{\circ} \mathrm{C}$ in refrigerator.

\section{Instruments}

The $\mathrm{pH}$ of the buffer was determined by PHS-3C pH Benchtop meter (INESA, Shanghai, China). UV-visible absorption spectra were recorded on a Hitachi U-1750 UV-vis spectrophotometer (Shimadzu, Japan). The fluorescence spectrum measurements were recorded on F-7100 (Hitachi, Tokyo, Japan). Temperature was controlled with an RCT basic package magnetic stirrer (IKA, Germany). A MIX-25P shaker (Miou, Hangzhou, China) was used to mix the solutions. All the photographs in the experiment were taken by an EOS M3 Digital Single Lens Reflex (Canon, Japan) with transmitted ultraviolet irradiation. The morphology of the CdTe QDs was recorded by JEM-2100F transmission electron microscopy (TEM).

\section{Synthesis of quantum dots}

The general procedure for the synthesis of CdTe quantum dots was as follows. Typically, $200 \mu \mathrm{L}$ L-cysteine $(100 \mu \mathrm{M})$ was added to a solution of $200 \mu \mathrm{L}$ MOPS buffer $\left(10 \mathrm{mM}, 150 \mathrm{mM} \mathrm{NaNO}_{3}\right.$, pH 7.6). Then, $200 \mu \mathrm{L} \mathrm{CdCl}_{2} \cdot 2.5 \mathrm{H}_{2} \mathrm{O}(2 \mathrm{mM}), 200 \mu \mathrm{L} \mathrm{Na}_{2} \mathrm{TeO}_{3}$ $(0.4 \mathrm{mM})$ and $20 \mu \mathrm{L} \mathrm{NaBH}_{4}(60 \mathrm{mM})$ were added in order into the above solution. Finally, after incubating for $75 \mathrm{~min}$ at $100{ }^{\circ} \mathrm{C}$, the CdTe quantum dots were obtained.

\section{Target DNA sensing}

In a typical assay, $40 \mu \mathrm{L}$ of target DNA of different concentrations, $40 \mu \mathrm{L} \mathrm{H} 1$ DNA $(1.25 \mu \mathrm{M})$ and $40 \mu \mathrm{L} \mathrm{H} 2$ DNA $(1.25 \mu \mathrm{M})$ were first mixed with MOPS buffer $(200 \mu \mathrm{L}, \mathrm{pH} 7.4,10 \mathrm{mM})$ and incubated at room temperature for $90 \mathrm{~min}$ to complete the CHA signal amplification reaction, thus releasing the G-quadruplex sequence. Then, after introducing $15 \mu \mathrm{M}$ hemin $(10 \mu \mathrm{L})$ and $1 \mathrm{M} \mathrm{K}^{+}(10 \mu \mathrm{L})$ into the above solution, it was incubated for $15 \mathrm{~min}$ at room temperature to form the G-quadruplex-hemin$\mathrm{K}^{+}$complex. Then, $200 \mu \mathrm{L}$ of $\mathrm{L}$-cysteine $(100 \mu \mathrm{M})$ was added into the above solution to initiate the oxidation reaction, which took place over $45 \mathrm{~min}$ at room temperature. Finally, $200 \mu \mathrm{L} \mathrm{CdCl}_{2}$ $\cdot 2.5 \mathrm{H}_{2} \mathrm{O}(2 \mathrm{mM}), 200 \mu \mathrm{L} \mathrm{Na}_{2} \mathrm{TeO}_{3}(0.4 \mathrm{mM})$ and $20 \mu \mathrm{L} \mathrm{NaBH}_{4}(60$ $\mathrm{mM})$ were sequentially added into the resulting solution. After incubation for $75 \mathrm{~min}$ at $100{ }^{\circ} \mathrm{C}$, the final solution was cooled to room temperature. The fluorescence spectra of the mixtures were measured on the spectrophotometer with a $365 \mathrm{~nm}$ excitation wavelength.

\section{Pretreatment of human serum samples}

Serum samples were taken and centrifuged for 15 min (8000 $\mathrm{rpm}$ ) using an ultrafiltration centrifuge tube (MWCO $=3 \mathrm{kDa}$ ). Then, the ultrafiltrates were diluted 100 times by ultrapure water and stored in $4{ }^{\circ} \mathrm{C}$ before analysis.

\section{Results and discussion}

\section{Sensing principle}

The principle of this G-quadruplex-controlled L-cysteine oxidation and CHA-assisted signal amplification fluorescence detection of DNA is elucidated in Scheme 1. The proposed strategy for the DNA assay included the following three steps: (1) CHA- 
Table 1 Sequences of oligonucleotides used in the study

Name $\quad$ Sequence $\left(5^{\prime}-3^{\prime}\right)$

G1 (G-quadruplex)

Target DNA

H1 (hairpin probe 1)

$\mathrm{H} 2$ (hairpin probe 2)

Single-base mismatch

Two-base mismatch

Non-complementary
GGGTTTTGGGTTTTGGGTTTTGGG

AGTCAGTGTGGAAAATCTCTAGC

GCTAGAGATTTTCCACACTGACTTCTCTAGCGGGTTTTGGGTTTTAGTCAGTGTGGAAAA

CTGACTAAAACCCAAAACCCGCTAGAGAAGTCAGTGTGGAAAATCTCTAGCGGGTTTTGGGTTTTGGGTTTTGGG AGTCAGTGTGGTAAATCTCTAGC

AGTCAGTCTGGAAATTCTCTAGC GCATGTATATTCTTCATCAATAG

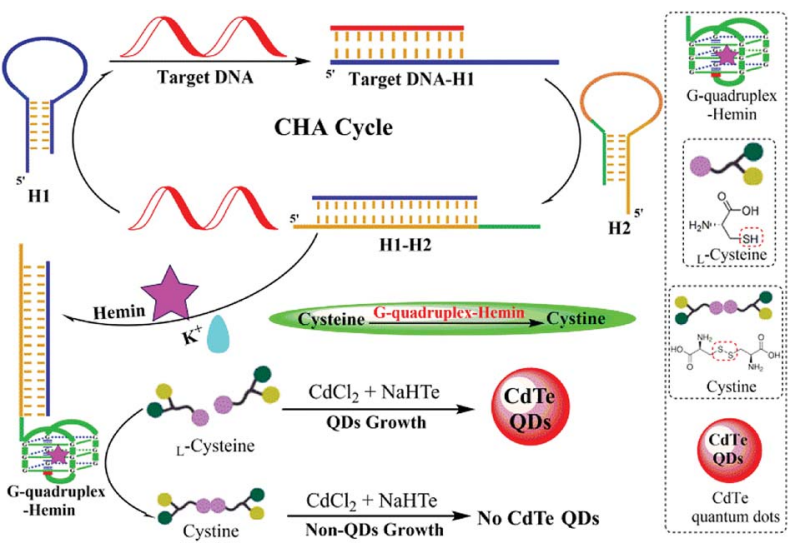

Scheme 1 Schematic illustration of the label-free and homogeneous fluorescence DNA assay based on the G-quadruplex-controlled oxidation of $\mathrm{L}$-cysteine and $\mathrm{CHA}$-assisted signal amplification.

assisted signal amplification triggered by target DNA, which releases the free G-quadruplex sequences, (2) the addition of hemin and $\mathrm{K}^{+}$to form the G-quadruplex-hemin- $\mathrm{K}^{+}$complex to initiate the following oxidation reaction of $\mathrm{L}$-cysteine to cystine, (3) synthesis of fluorescent CdTe QDs by adding the $\mathrm{CdCl}_{2}$, $\mathrm{Na}_{2} \mathrm{TeO}_{3}$ and $\mathrm{NaBH}_{4}$ into the resulting aforementioned solution. Two types of DNA hairpin probes were ingeniously designed and employed in this system, including $\mathrm{H} 1$ and $\mathrm{H} 2$ (hairpin probes, shown in Table 1). The $\mathrm{H} 1$ and $\mathrm{H} 2$ probes could form a stem-loop structure due to the binding of the complementary sequences at the ends. $\mathrm{H} 2$ was the signal hairpin composed of a G-quadruplex sequence. When $\mathrm{H} 2$ retains its hairpin structure, the segment of the G-quadruplex sequence is hidden in the stem. Thus, the G-quadruplex cannot form which decreases the background CHA. Additionally, the sequence of the $\mathrm{H} 1$ is partially complementary to the target DNA, while the entire sequence of $\mathrm{H} 1$ is complementary to $\mathrm{H} 2$. When the $\mathrm{H} 1$ was confronted with target DNA, the recognition between the target DNA and $\mathrm{H} 1$ would break the hairpin structure, initiating the first branch migration and the opening of H1. Then, the newly exposed sequence in the opened $\mathrm{H} 1$ serves as a new trigger which hybridizes with $\mathrm{H} 2$ and initiates the second branch migration, resulting in the formation of an $\mathrm{H} 1-\mathrm{H} 2$ complex and the release of the G-quadruplex sequences. Finally, the released G-quadruplex has the capability to bind with hemin and act as an HRP-like DNAzyme for catalyzing L-cysteine oxidation, and L-cysteine serves as a ligand to mediate biomimetic synthesis of luminescent quantum dots. Hence, the degree of inhibition for the QDs synthesis is proportional to the concentration of target DNA. In this way, the target DNA trigger catalyzes the autonomous assembly and generation of numerous $\mathrm{H} 1-\mathrm{H} 2$ complexes and G-quadruplexes, which are exponentially produced. Therefore, the basis of the L-cysteinemediated synthesis of fluorescent CdTe QDs and CHA-assisted target recycling strategy provides the possible high sensitivity for the assay of nucleic acids through the turn-off mode.

\section{Feasibility of the fluorescence DNA detection}

Fluorescence experiments were performed to investigate the feasibility of the G-quadruplex-hemin- $\mathrm{K}^{+}$-controlled oxidation of L-cysteine and CHA-assisted signal amplification triggered by target nucleic acids.

The ability of G-quadruplex-hemin- $\mathrm{K}^{+}$to catalyze the oxidation of L-cysteine to cystine, and L-cysteine to serve as the ligand for CdTe QDs synthesis was affirmed first. Evidently, in the absence of the G-quadruplex-hemin- $\mathrm{K}^{+}$complex, L-cysteine could not be oxidized and still maintained the capability to assist CdTe QDs synthesis. As the TEM image shows, the CdTe QDs which used L-cysteine as a ligand are quasi-spherical and non-aggregated with an average diameter of $\sim 4 \mathrm{~nm}$ (Fig. 1A). ${ }^{29}$ As shown in Fig. 1B, the characteristic absorption peak at
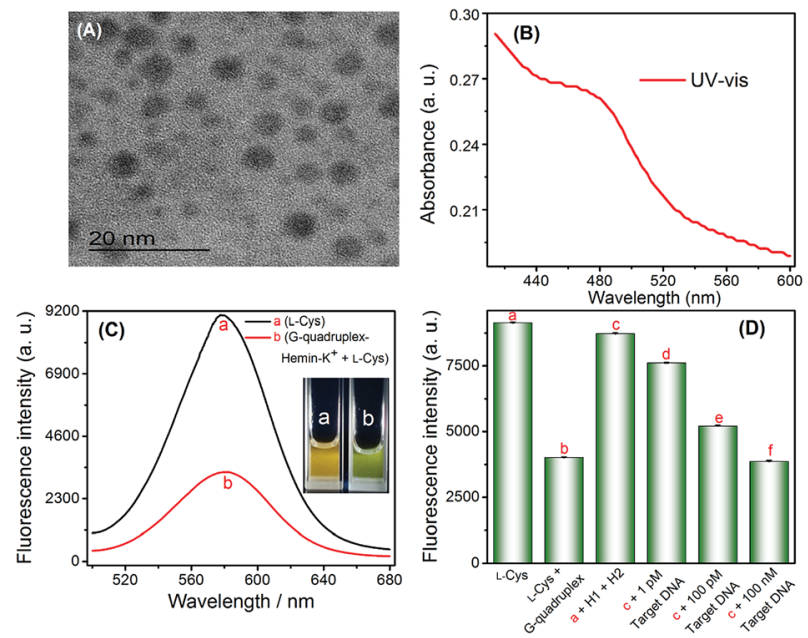

Fig. 1 (A) TEM of CdTe QDs, (B) UV-vis spectrum of CdTe QDs, (C) fluorescence spectrum under different conditions. (a) L-Cys only; (b) G-quadruplex-hemin- $\mathrm{K}^{+}+\mathrm{L}-\mathrm{Cys}$, (D) experimental feasibility verification under different conditions. 
approximately $480 \mathrm{~nm}$ was found in the UV-vis spectra. Furthermore, a significant fluorescence signal was acquired (as shown in Fig. 1C-a, and inset (a)). However, in the presence of the G-quadruplex-hemin- $\mathrm{K}^{+}$complex, L-cysteine was effectively converted into cystine via the catalyzed oxidation reaction, which led to a much lower fluorescence intensity (shown in Fig. 1C-b, and inset (b)).

To investigate the feasibility of the proposed strategy towards target HIV DNA detection, fluorescence spectra signals of the sensing system under various conditions were recorded and displayed in Fig. 1D. Obviously, a relatively weak fluorescence signal (Fig. 1D-b) was observed when G1 (G-quadruplex sequence, Table 1) was pre-incubated with L-cysteine in the presence of hemin and $\mathrm{K}^{+}$(b vs. a). No significant fluorescence signal changes were observed when $\mathrm{H} 1$ and $\mathrm{H} 2$ DNA (in the absence of a target DNA) were added into L-cysteine (solution a), which indicated that the formation of the G-quadruplex structure is effectively suppressed by the formation of the $\mathrm{H} 2$ hairpin structure, and $\mathrm{H} 1$ and $\mathrm{H} 2$ can stably coexist in solution. In contrast, in the presence of the target DNA, the fluorescence intensity of CdTe QDs considerably decreased (c vs. d-f). Such a decrease in the fluorescence signal is due to the fact that the target DNA binds and opens the $\mathrm{H} 1$ and $\mathrm{H} 2$ hairpin structure, leading to the release of G-quadruplex sequence, and then causes the formation of the G-quadruplex-hemin- $\mathrm{K}^{+}$complex; this complex oxidizes L-cysteine to cystine, thus inhibiting the formation of quantum dots. These experimental results clearly demonstrated that the fluorescence detection of HIV DNA could be readily achieved using the proposed system.

\section{Optimization of experimental conditions}

Before the standard DNA assay, some important parameters were optimized to obtain the best sensing performance, including the conditions of CdTe QDs synthesis, CHA-assisted signal amplification reaction, formation of the G-quadruplexhemin $-\mathrm{K}^{+}$complex, and the oxidation of $\mathrm{L}$-cysteine to cystine, etc. As shown in Fig. $\mathrm{S} 1 \mathrm{~A}$ and $\mathrm{S} 1 \mathrm{~B}, \dagger$ the maximum fluorescence signals were obtained at $100{ }^{\circ} \mathrm{C}$, and the peak fluorescent intensity was achieved at the time of $75 \mathrm{~min}$ for CdTe QDs synthesis (Fig. S1C and $\mathrm{S} 1 \mathrm{D}^{\dagger}$ ). Therefore, the conditions of 75 min incubation at $100{ }^{\circ} \mathrm{C}$ were chosen to synthesize CdTe QDs. As shown in Fig. S2 and $\mathrm{S} 3, \uparrow$ the maximum fluorescence value obtained at the concentrations of L-cysteine and $\mathrm{NaBH}_{4}$ were $100 \mu \mathrm{M}$ and $60 \mathrm{mM}$, respectively. The CHA-assisted signal amplification reaction conditions mainly involve the concentrations of $\mathrm{H} 1$ and $\mathrm{H} 2$, and the total reaction time after adding target DNA into blank solution. As shown in Fig. S4 and S5, $\uparrow$ the concentrations of $\mathrm{H} 1$ and $\mathrm{H} 2$ at $1.25 \mu \mathrm{M}$, and reaction time of 90 min were used in the subsequent experiments, respectively. The formation of the G-quadruplex-hemin- $\mathrm{K}^{+}$complex and oxidation L-cysteine to cystine is crucial for the entire experiment. The concentration of $\mathrm{K}^{+}$and hemin were investigated subsequently, respectively. As shown in Fig. S6 and Fig. S7, $\dagger$ the maximum fluorescence difference values were obtained at the concentrations of $10 \mathrm{mM} \mathrm{K} \mathrm{K}^{+}$and $15 \mu \mathrm{M}$ hemin. The time of $15 \mathrm{~min}$ for the formation the G-quadruplex-hemin- $\mathrm{K}^{+}$complex
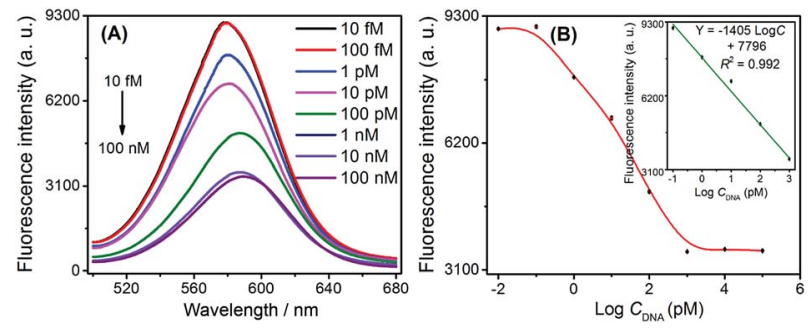

Fig. 2 Analytical performance of the proposed label-free and homogeneous strategy for DNA detection. (A) Fluorescence spectra of obtained CdTe QDs in the presence of various DNA concentrations. (B) Calibration curve for DNA detection. Inset: the linearity of fluorescence intensity with respect to logarithmic DNA concentrations ranging from $0.1 \mathrm{pM}$ to $1 \mathrm{nM}$. Error bars were estimated from three replicate measurements.

after added the hemin and $\mathrm{K}^{+}$, and $45 \mathrm{~min}$ for the oxidation of $\mathrm{L}^{-}$ cysteine to cystine by the G-quadruplex-hemin complex was sufficient (as shown in Fig. S8 and S9†).

\section{Analytical performance of the DNA sensor}

Under the optimal conditions, we used this G-quadruplexcontrolled oxidation of L-cysteine and L-cysteine-mediated synthesis of fluorescent quantum dots system to detect synthetic HIV DNA by monitoring fluorescent signals. A series of fluorescence spectra are shown in Fig. 2A. The fluorescence intensity of CdTe QDs gradually decreased in conjunction with increasing concentrations of target DNA from $10 \mathrm{fM}$ to $100 \mathrm{nM}$. It could be seen that the fluorescence intensity of CdTe QDs and the logarithmic concentration of HIV DNA showed a good linear relationship within the range from $0.1 \mathrm{pM}$ to $1 \mathrm{nM}$ (as shown in the inset of Fig. 2B). The linear equation was found to be $Y=$ $-1405 \log C+7796$, with the correlation coefficient $R^{2}=0.992$, and the detection limit was $0.12 \mathrm{pM}(3 \sigma)$. The reproducibility expressed as relative standard deviation (RSD) of $10 \mathrm{pM}$ target DNA was $4.8 \%$. This high sensitivity could be ascribed to the effective signal amplification of the developed enzyme-free CHA-assisted signal amplification strategy.

The analytical performance of this assay was further compared with several other reported methods and summarized in Table S1. $\uparrow$ Compared to the previous enzyme-involved and enzyme-free protocols, the proposed method exhibited comparable sensitivity with a simpler operation and design.

\section{Specific discrimination for base mismatches}

The capability to specifically detect base mismatches was investigated. The selectivity of the sensing scheme was also evaluated by monitoring the fluorescence response of CdTe QDs when this method was challenged with one base- and two basemismatches, and the non-complementary DNA as well as perfectly complementary DNA sequences at same concentration of 1 nM. Fig. 3A displayed an obvious fluorescence signal decreased with one base- and two base-mismatches as well as fully complementary DNA sequences, respectively. In contrast to the significant reduction of fluorescence intensity caused by the target DNA, the addition of the non-complementary and 

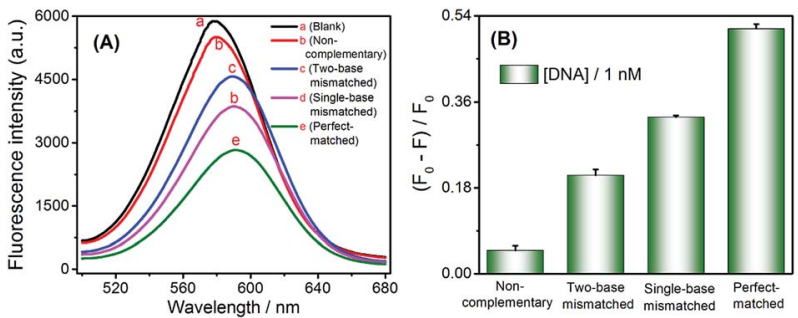

Fig. 3 Nucleic acid sequence selectivity of the proposed label-free and homogeneous strategy using different DNA targets at same concentration of $1 \mathrm{nM}$. (A) Fluorescence spectra of obtained CdTe QDs. (B) The $\left(F_{0}-F\right) / F_{0}$ in the presence of target DNA with different base mismatches. $F_{0}$ and $F$ represent the fluorescence signal of the solution containing $\mathrm{H} 1$ and $\mathrm{H} 2$ in the absence and presence of $1 \mathrm{nM}$ target DNA, respectively.

Table 2 The detection of target DNA in spiked human serum samples

\begin{tabular}{llccl}
\hline Sample & Added, pM & Found $^{a}, \mathrm{pM}$ & Recovery $^{b}, \%$ & RSD $^{c}, \%$ \\
\hline \multirow{2}{*}{ \% serum } & 1.00 & $0.93 \pm 0.05$ & 93 & 6.2 \\
& 10 & $9.6 \pm 0.15$ & 96 & 3.8 \\
& 100 & $104 \pm 5$ & 104 & 4.1 \\
& 1000 & $997 \pm 18$ & 99 & 3.3
\end{tabular}

${ }^{a}$ Mean and standard deviation of results $(n=3) .{ }^{b}$ Recovery $(\%)=$ $\left(C_{\text {Found }} / C_{\text {Added }}\right) \times 100 \%{ }^{c}$ Relative standard deviation.

base-mismatched strands triggered a less reduction in fluorescence intensity (as shown in Fig. 3B). These results demonstrated that this method can distinguish single-base mismatches in DNA sequences.

\section{Practical applicability}

To validate the application of this proposed DNA assay to real biological samples, human serum samples spiked with different concentrations of target HIV DNA were analyzed. To eliminate potential interferences, the serum samples were filtered using a centrifugal filtration device (MWCO $=3 \mathrm{kDa}$ ), which removed small molecules (e.g. GSH) and ions (e.g. $\left.\mathrm{K}^{+}\right)$. The resulting filtrate was diluted 100-fold before use. As demonstrated in Table 2, the spiked recovery of HIV DNA from the serum samples was $93-104 \%$, and the relative standard deviation (RSD) was as low as $3.3 \%$, which showed a strong consistency between the spiked concentrations. Therefore, these results demonstrated the tolerance of the proposed method to the complicated sample matrix of serum.

\section{Conclusions}

In summary, a well-designed synthetic fluorescent quantum dot-based novel turn-off mode strategy for the highly sensitive label-free detection of DNA was developed. This method mainly was based on the L-cysteine-modulated synthesis of CdTe QDs, the horseradish peroxidase-mimicking oxidative control of the L-cysteine to cystine conversion performed by G-quadruplexhemin- $\mathrm{K}^{+}$, and the CHA-assisted signal amplification. Benefitting from the CHA-assisted signal amplification, and the intrinsically high sensitivity of fluorescence detection, this system revealed good performance in nucleic acid analysis with a detection limit of $0.12 \mathrm{pM}$. This method is more straightforward and cost-effective than other current analytical methods, and this is the first example showing the technique of biomimetic synthesis of luminescent nanomaterials coupled with nucleic acid signal amplification to directly monitor DNA. ${ }^{30}$ Also, considering the versatility of aptamers or peptides as recognition ligands, the proposed biomolecular-modulated quantum dot synthesis-based DNA bioassay platform is expected to have a broad spectrum of applications in bioanalysis. ${ }^{31,32}$

\section{Conflicts of interest}

There are no conflicts to declare.

\section{Acknowledgements}

This work was supported by the National Key Research and Development Program of China (Grant No. 2016YFA0201400 and No. 2016YFC1200300).

\section{Notes and references}

1 S. K. Arya and S. Bhansali, Chem. Rev., 2011, 111, 6783-6809. 2 J. Bell, Nature, 2004, 429, 453-456.

3 P. P. Liang, J. Canoura, H. X. Yu, O. Alkhamis and Y. Xiao, ACS Appl. Mater. Interfaces, 2018, 10, 4233-4242.

4 W. Li, C. X. Yang and X. P. Yan, Chem. Commun., 2017, 53, 11469-11471.

5 N. X. Li, J. Zheng, C. R. Li, X. X. Wang, X. H. Ji and Z. K. He, Chem. Commun., 2017, 53, 8486-8488.

6 P. Zhang, J. Jiang, R. Yuan, Y. Zhuo and Y. Q. Chai, J. Am. Chem. Soc., 2018, 140, 9361-9364.

7 X. Y. Wang, F. Chen, D. X. Zhang, Y. Zhao, J. Wei, L. H. Wang, S. P. Song, C. H. Fan and Y. X. Zhao, Chem. Sci., 2017, 8, 4764-4770.

8 Y. D. Sun, P. Peng, R. Y. Guo, H. H. Wang and T. Li, Biosens. Bioelectron., 2018, 104, 32-38.

9 Y. Zhao, H. M. Wang, W. Tang, S. C. Hu, N. Li and F. Liu, Chem. Commun., 2015, 51, 10660-10663.

10 P. P. Chen, P. Wu, J. B. Chen, P. Yang, X. F. Zhang, C. B. Zheng and X. D. Hou, Anal. Chem., 2016, 88, 2065-2071.

11 R. Liu, C. Q. Wang, Y. M. Xu, J. Y. Hu, D. Y. Deng and Y. Lv, Anal. Chem., 2017, 89, 13269-13274.

12 B. Y. Guo, Y. Y. Sheng, K. Zhou, Q. S. Liu, L. Liu and H. C. Wu, Angew. Chem., Int. Ed., 2018, 57, 3602-3606.

13 N. Goswami, F. X. Lin, Y. B. Liu, D. T. Leong and J. P. Xie, Chem. Mater., 2016, 28, 4009-4016.

14 Y. F. Kong, J. Chen, H. W. Fang, G. Heath, Y. Wo, W. L. Wang, Y. X. Li, Y. Guo, S. D. Evans, S. Y. Chen and D. J. Zhou, Chem. Mater., 2016, 28, 3041-3050.

15 Y. Hu, Z. Y. Miao, M. J. Zhang, X. T. Yang, Y. Y. Tang, S. Yu, C. X. Shan, H. M. Wen and D. Zhu, Anal. Chem., 2018, 90, 5678-5686. 
16 K. Huang, K. L. Xu, J. Tang, L. Yang, J. R. Zhou, X. D. Hou and C. B. Zheng, Anal. Chem., 2015, 87, 6584-6591.

17 Y. F. Chen, Z. Chen, Y. J. He, H. L. Lin, P. T. Sheng, C. B. Liu, S. L. Luo and Q. Y. Cai, Nanotechnology, 2010, 21, 125502.

18 A. Niazov-Elkan, E. Golub, E. Sharon, D. Balogh and I. Willner, Small, 2014, 10, 2883-2891.

19 H. Y. Li, J. F. Chang, P. P. Gai and F. Li, ACS Appl. Mater. Interfaces, 2018, 10, 4561-4568.

20 F. A. Wang, X. Q. Liu, C. H. Lu and I. Willner, ACS Nano, 2013, 7, 7278-7286.

21 Y. He, D. L. Chen, M. X. Li, L. Fang, W. J. Yang, L. J. Xu and F. F. Fu, Biosens. Bioelectron., 2014, 58, 209-213.

22 D. F. Wang, C. L. Vietz, T. Schroeder, G. Acuna, B. Lalkens and P. Tinnefeld, Nano Lett., 2017, 17, 5368-5374.

23 X. L. Zuo, F. Xia, Y. Xiao and K. W. Plaxco, J. Am. Chem. Soc., 2010, 132, 1816-1818.

24 B. C. Yin, Y. Q. Liu and B. C. Ye, J. Am. Chem. Soc., 2012, 134, 5064-5067.
25 J. Huang, Y. R. Wu, Y. Chen, Z. Zhu, X. H. Yang, C. Y. J. Yang, K. M. Wang and W. H. Tan, Angew. Chem., Int. Ed., 2011, 50, 401-404.

26 P. P. Chen, P. Wu, Y. X. Zhang, J. B. Chen, X. M. Jiang, C. B. Zheng and X. D. Hou, Anal. Chem., 2016, 88, 1238612392.

27 H. M. Fang, N. L. Xie, M. Ou, J. Huang, W. S. Li, Q. Wang, J. B. Liu, X. H. Yang and K. M. Wang, Anal. Chem., 2018, 90, 7164-7170.

28 Y. Jiang, B. L. Li, J. N. Milligan, S. Bhadra and A. D. Ellington, J. Am. Chem. Soc., 2013, 135, 7430-7433.

29 J. b. Liu, X. H. Yang, K. M. Wang, X. X. He, Q. Wang, J. Huang and Y. Liu, ACS Nano, 2012, 6, 4973-4983.

30 X. W. He and N. Ma, Small, 2013, 9, 2527-2531.

31 H. M. Meng, H. Liu, H. L. Kuai, R. Z. Peng, L. T. Mo and X. B. Zhang, Chem. Soc. Rev., 2016, 45, 2583-2602.

32 Y. Cheng, C. L. Sun, X. W. Ou, B. F. Liu, X. D. Lou and F. Xia, Chem. Sci., 2017, 8, 4571-4578. 